\title{
3'-End cDNA Pool Suitable for Differential Display from a Small Number of Cells
}

BioTechniques 24:842-852 (May 1998)

\author{
S. Zhao, G. Molnar, J. Zhang, \\ L. Zheng, L. Averboukh and \\ A.B. Pardee \\ Dana-Farber Cancer Institute, \\ Boston, MA, USA
}

\section{INTRODUCTION}

The differential display (DD) technique has already become a basic molecular biology method used to address questions of gene expression in a variety of biological systems (9). There is a need to adapt DD to samples with limited RNA content; for example, low numbers of cells captured mechanically or by microdissection from clinical needle biopsy and from embryonic or neural cells. The original system for extensive DD utilized 10-100 $\mu \mathrm{g}$ of total cellular RNA, requiring more than 500000 cells, assuming that one cell has 20 pg total RNA (7). Therefore, we have developed a new primer combination to construct inexhaustible $3^{\prime}$ cDNA pools by reverse transcription polymerase chain reaction (RT-PCR) from only 1000 or fewer cells. This pool is suitable for DD and for amplification of specific genes.

Construction of libraries from small RNA samples by PCR-based cDNA amplification methods has been reported. Brady and Iscove established a cDNA library from a single cell by $3^{\prime}$ poly(A) tailing of the first-strand cDNA (3). The recently developed CapFinder ${ }^{\mathrm{TM}}$ cDNA Library Synthesis method (CLONTECH Laboratories, Palo Alto, CA, USA) provides another approach (4), which takes advantage of a primer switch at a $5^{\prime}$ deoxycytidine end for second-strand cDNA synthesis, resulting in large numbers of fully reverse-transcribed messages from small amounts of total cellular RNA (0.1-0.2 $\mu \mathrm{g})$. These techniques have not yet been combined with DD. Also, they in- clude two difficulties related to the synthesis of full-length cDNAs. One is that reverse transcriptase cannot usually transcribe an entire mRNA sequence. The second is the disproportionally lesser amplification of long fragments compared with shorter ones. Our approach avoids these problems by using primers that generate a more uniformly sized cDNA pool (about 100-800 bp), which should not be subject to PCRsize bias and hence will produce representative DD patterns.

To generate a representative $3^{\prime}$ cDNA pool, we designed primers with one degenerate base at the $3^{\prime}$ end of the anchor and four degenerate bases at the $3^{\prime}$ end of the arbitrary primer. Also, these primers are longer (28-mers) than ones used initially for DD (10-16mers). These extended primers greatly increase the reproducibility of DD (13), but they also decrease the number of resulting bands. To increase this number, arbitrary ends were built onto the original short primers. The sequence of these ends takes advantage of the mismatch tolerance of Klenow DNA polymerase at low-annealing temperature for the second-strand synthesis and increases the binding frequency of the primers. Restriction sites placed within the primers are useful for making hybridization probes and cloning.

The cDNA pool produced by these degenerate primers reproducibly contained sense DNA fragments, as demonstrated by finding prostate-specific antigen (PSA), S100P (1), maspin (12), thymidine kinase and cyclin A sequences in them. We showed that the pool contains cDNAs from rare mes- 
Table 1. Construction of $3^{\prime}$-End cDNA Libraries

\section{Step 1. Reverse Transcription}

The usual RT reaction contained 0.1-0.2 $\mu \mathrm{g}$ total RNA with $1 \mu \mathrm{M}$ degenerate anchor primer H-T14V (Table 2), 1× SuPERSCRIPT TM buffer (50 mM Tris- $\mathrm{HCl}, \mathrm{pH} 8.3,75 \mathrm{mM} \mathrm{KCl}, 3 \mathrm{mM} \mathrm{MgCl}$ ), $1 \mathrm{mM}$ dithiothreitol (DTT) and $250 \mu \mathrm{M}$ dNTPs in $20 \mu \mathrm{L}$ final volume. This reaction mixture was first preheated to $65^{\circ} \mathrm{C}$ for $5 \mathrm{~min}$, followed by a 5 -min incubation at $37^{\circ} \mathrm{C}$. Then, $200 \mathrm{U}$ SUPERSCRIPT II Reverse Transcriptase (Life Technologies) in $1 \mu \mathrm{L}$ were added, and the mixture was further incubated for 50 $\min$ at $37^{\circ} \mathrm{C}$. The reaction was stopped by heat-inactivation of the enzyme at $95^{\circ} \mathrm{C}$ for 5 min then quickly chilled on ice.

\section{Step 2. Second-Strand cDNA Synthesis}

Five microliters of first-strand mixture were supplemented with $250 \mu \mathrm{M}$ dNTPs and $1 \times$ Klenow reaction buffer $(50 \mathrm{mM}$ Tris- $\mathrm{HCl}$, $\mathrm{pH} 8.0,10 \mathrm{mM} \mathrm{MgCl}_{2}, 50 \mathrm{mM} \mathrm{NaCl}$ ) in $20 \mu \mathrm{L}$ of final volume. The second-strand cDNA was then synthesized by Klenow enzyme (3.65 U/reaction; Life Technologies) with the degenerate primer DNP256 $(1 \mu \mathrm{M})$. The reaction was incubated at $37^{\circ} \mathrm{C}$ for $45 \mathrm{~min}$ and stopped by incubation at $65^{\circ} \mathrm{C}$ for $10 \mathrm{~min}$.

\section{Step 3. Amplification of Double-Stranded cDNAs}

To create a cDNA library, one-fifth of the second-strand reaction mixture was used in the presence of $1 \mu \mathrm{M} \mathrm{H}$-T14 and DP256 primers, and $0.1 \mu \mathrm{M}$ dNTPs, together with $3.75 \mathrm{U}$ of the recombinant thermostable $\mathrm{r}$ Tth DNA Polymerase (Perkin-Elmer, Norwalk, CT, USA) per reaction in $20 \mu \mathrm{L}$. The PCR temperature profiles applied for 25 cycles on a TRIO-Thermoblock ${ }^{\circledR}$ Thermal Cycler (Biometra, Göttingen, Germany) were: $94^{\circ} \mathrm{C}$ for $45 \mathrm{~s}, 55^{\circ} \mathrm{C}$ for $2 \min$ and $65^{\circ} \mathrm{C}$ for $4 \mathrm{~min}$.

\section{Step 4. Purification of PCR Products}

To prepare for further steps (DD or primer-specific amplification), unincorporated primers and short fragments were removed

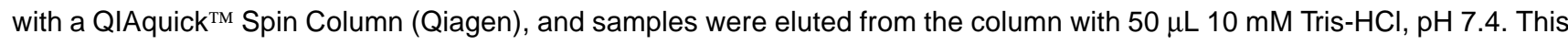
cleanup step should decrease the production of short primer dimers.

sages and that it can semiquantitively preserve abundance differences between RNA samples.

\section{MATERIALS AND METHODS}

\section{Preparation of Total RNA}

RNA was isolated from either cultured LNCaP or Jurkat cells or from anticoagulant-treated human peripheral blood (5), using the TRIzol ${ }^{\circledR}$ Reagent (Life Technologies, Gaithersburg, MD, USA). RNA was obtained from counted cells with the RNeasy ${ }^{\circledR}$ Mini Kit (Qiagen, Chatsworth, CA, USA) as suggested by the manufacturer.

\section{Construction of Degenerate 3 '-End cDNA Pools}

Figure 1 outlines the scheme of this method, and Table 1 describes the procedures. All concentrations are final ones. All primers were synthesized by Integrated DNA Technology (Coralville, IA, USA).

\section{Amplification by Specific Primers}

For PSA-specific amplification, 1 $\mu \mathrm{L}$ of the column-purified cDNA pool from Table 1, Step 4 was used as template with PSA primers: $\mathrm{AP}_{1405}$ (GCT TGG AGG ACT TCA ATA CAC C) and $\mathrm{SP}_{1086}$ (GGT GTC TGT GTT ATT TGT GGG G) or $\mathrm{SP}_{843}$ (CCC CCT ATT GTA GTA AAC TTG G). Maspin was amplified by the $\mathrm{MP}_{2354}$ (GAT TCA AGG CAT TGC TTC CTG) and MP $_{1935}$ (AAT CGC TGC CAT GCC ATC AAT) primers, and HSIX (2) was amplified by the homeobox primers $\mathrm{P} 1$ (GAG ACC AGC TAC TGG TTC AA) and P2 (GAG AGA GTT GGT TCT GCT TG). The PCR conditions for the rTth enzyme are: $94^{\circ} \mathrm{C} 45 \mathrm{~s}, 55^{\circ} \mathrm{C}$ for 2 $\min , 65^{\circ} \mathrm{C}$ for $6 \min$ (in 30 cycles) then finally $72^{\circ} \mathrm{C}$ for $7 \mathrm{~min}$.

\section{Differential Display}

The column-purified samples from Table 1, Step 4 were used as substrate for this assay. These samples were diluted 25 times, and then $2-\mu \mathrm{L}$ aliquots were processed for DD. Reaction volume $(20 \mu \mathrm{L})$ contained $2.5 \mu \mathrm{M}$ dNTPs, $0.4 \mu \mathrm{M}$ upstream and downstream primers, chosen from N1-TC, N1-AG, $\mathrm{N} 1-\mathrm{TG}$ and N1-CC (N1 = 5'-GAC CTC
TAG ATG CTG TTG) and N2-T14-CT, N2-T14-CA and N2-T14-GT (N2 = 5' TGC GAA GC) or HT11-A and AP1 primer supplied in the RNAimage ${ }^{\mathrm{TM}}$ kit 1 (GenHunter, Nashville, TN, USA). Three microcuries [33P]dATP (NEN Life Science Products, Boston, MA, USA) and 1 U Taq DNA Polymerase (Perkin-Elmer) were included in the reaction. The PCR conditions were as follows: $94^{\circ} \mathrm{C}$ for $15 \mathrm{~s}, 60^{\circ} \mathrm{C}$ for $30 \mathrm{~s}$ and $72^{\circ} \mathrm{C}$ for 1 min during 30 cycles, with 5 -min extension at $72^{\circ} \mathrm{C}$. Then DD was carried out as described (8).

\section{Northern Hybridization}

Twenty micrograms total RNA were separated on a formaldehyde-agarose gel and then transferred onto nitrocellulose membranes. The blots were hybridized with [32P]dCTP-labeled probes as described (10).

\section{Southern Hybridization}

The total PCR products were separated on a $1 \%$ agarose gel then transferred onto nitrocellulose membranes. The probe was random-prime-labeled by [ $\left.{ }^{32} \mathrm{P}\right] \mathrm{dCTP}$ as described (11). 


\section{Research Reports}

\section{RESULTS}

\section{Design of Primers to Construct a 3'-End cDNA Library by Random Priming}

To design primers for creating a general cDNA pool from small samples, three aims were taken into account. First, the primers should initiate synthesis of the entire cDNA population without distinguishing between frequent and rare genes. For this purpose, primers with degenerate $3^{\prime}$ ends were chosen to initiate synthesis of both cDNA strands. Second, to reduce the false-positive bands and increase reproducibility of the results, longer primers, such as those applied for DD or PCR fingerprinting (13), were designed and used at higher temperature. Third, for further manipulations, such as cloning, the asymmetric restriction sites HindIII and XbaI were inserted into the middle of the $3^{\prime}$ and $5^{\prime}$ primers, respectively.

\section{3'Anchor Primer (H-T14V)}

The poly(A) tail of eukaryotic mRNAs is usually selected for reverse transcriptase reactions using different lengths of oligo(dT) primers with or without additional flanking regions. Similarly, our 3' primer consists of 14 (dT) and therefore anneals at the $3^{\prime}$ end of poly $\left(\mathrm{A}^{+}\right)$mRNAs. It also contains one degenerate base ( $\mathrm{V}=\mathrm{A}, \mathrm{C}$ or $\mathrm{G})$ as an anchor at its $3^{\prime}$ end, and the restriction site HindIII in the center, which overlaps with two dT bases (Table 2, underlined). Finally, the $5^{\prime}$ end of the primer was supplemented with nine GC-rich, arbitrarily selected bases, resulting in 28-base total length. For the reverse transcriptase reaction, RNA from about 2500 cells was initially used (Table 1, Step 1).

\section{5' Degenerate Primer (DNP256)}

The $5^{\prime}$ primer was designed to anneal within $600 \mathrm{bp}$ of the 3' priming site of the first-strand cDNA for the entire population of sequences. At least a 10-mer is required to get reproducible DD patterns when the annealing temperature is low $\left(40^{\circ} \mathrm{C}\right)(6)$, and the resulting DD pattern contains about 100 bands, corresponding to only a few per- cent of the first-strand cDNAs. Following the same strategy as described above, the four $3^{\prime}$ arbitrary bases were replaced by degenerate bases (NNNN), thereby increasing the probability of binding by 256 times. Together with rare random mismatches introduced during the subsequent PCRs, this frequent binding should result in too many amplified fragments that are too short. Therefore, an additional 10 arbitrarily selected $5^{\prime}$ bases and the XbaI restriction site (Table 2, underlined), which overlapped with two bases of the original 10-mer, were added to make a 28-mer primer. To produce sufficient fragments with the DNP256 primer, a low-stringency step $\left(37^{\circ} \mathrm{C}\right)$ with Klenow enzyme was used for second-strand cDNA synthesis (Table 1 , Step 2) in place of $\mathrm{r} T \mathrm{th}$, which infrequently produces mismatches. The an- nealing temperature was raised during subsequent cycles, and rTth and nondegenerate primers were used to decrease mismatches, retain the desired primerbinding frequency and increase the fragment size to $100-800 \mathrm{bp}$.

\section{Construction of $3^{\prime}$-End cDNA Pools}

cDNAs were amplified by 25 PCR cycles with H-T14 and DP256 primers (Table 2), which lack the degenerate $3^{\prime}$ ends (V or NNNN) and whose specificities depend on the remaining arbitrary sequences (Table 1, Step 3). This procedure was tested for production of 3 -end cDNA pools from three different RNA samples. Two were from PSApositive and -negative human white blood cells (WBC), and the third was from LNCaP cells, which express PSA. The amplifed products were visualized

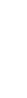


Table 2. Primer Sequences

\begin{tabular}{|c|c|}
\hline H-T14V & $\begin{array}{l}\text { 5'-CCT CCT GCG AAG CTT TTT TTT TTT TTT Va } \\
\text { (28-mer) }\end{array}$ \\
\hline H-T14 & $\begin{array}{l}\text { 5'-CCT CCT GCG AAG CTT TTT TTT TTT TTT } \\
\text { (27-mer) }\end{array}$ \\
\hline DNP256 & $\begin{array}{l}\text { 5'-GTT CGA GAC CTC TAG ATG CTG TTG NNN Nb } \\
\text { (28-mer) }\end{array}$ \\
\hline DP256 & $\begin{array}{l}\text { 5'-GTT CGA GAC CTC TAG ATG CTG TTG } \\
\text { (24-mer) }\end{array}$ \\
\hline \multicolumn{2}{|c|}{$\mathrm{aV}=\mathrm{G}, \mathrm{A}, \mathrm{C}$} \\
\hline
\end{tabular}

on an ethidium bromide-stained agarose gel (Figure 2A). The size distribution of products ranged between 100 and $800 \mathrm{bp}$, as desired.

Several variations of the procedure described above were tested. Production of cDNA pools using polymerases rTth, Taq and Vent ${ }_{R}^{\circledR}$ was compared using the same PCR conditions. All produced comparable pools, indicating that the method is reproducible and that any of these DNA polymerases can be used (data not shown). The cDNA pool was also enriched by a second amplification $(2 \times 25$ cycles $)$, using the same primers and similar PCR conditions as in the first run, except that the extension time was 6 min vs. 4 min. This procedure efficiently amplified fragments up to $1 \mathrm{~kb}$ (Figure 2B), which were faint on agarose gels after the first PCR. However, the use of $2 \times 25$ cycles did not significantly increase the quality of DD patterns, and so in future experiments, we used only $1 \times 25$ PCR cycles.

\section{The 3'-End cDNA Pool Can Supply Templates for Differential Display}

A principle application of $3^{\prime}$-end cDNA pools is for the identification of differentially expressed genes. First, we compared the DD pattern obtained without amplification to that produced from a $3^{\prime}$ cDNA pool. Double-stranded

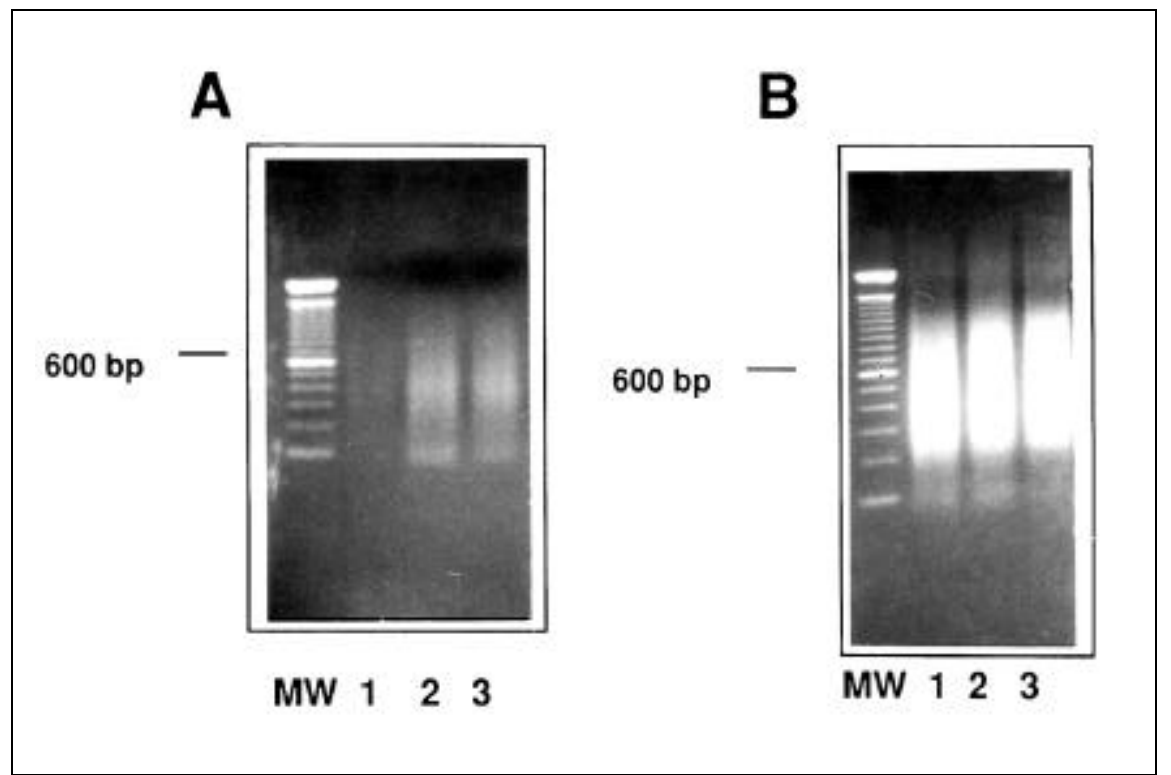

Figure 2. Degenerate primers produce a cDNA library. (A) The same amounts of total RNA $(0.1 \mu \mathrm{g})$ isolated from PSA-negative WBC (lane 1), PSA-positive WBC (lane 2) and exponentially growing LNCaP cells (lane 3 ) were reverse-transcribed with $\mathrm{H}-\mathrm{T} 14 \mathrm{~V}$ primer. After second-strand synthesis with DNP256 primer, cDNA libraries were constructed by 25 amplifications with H-T14 and DP256 primers. (B) Samples from Panel A were subjected to a second amplification with the same second primer set. 


\section{Research Reports}

cDNAs from LNCaP cells were prepared using H-T14V and DNP256 primers. Before pool amplification, we applied the DD technique with a pair of nested primers, N2-T14-GT and N1TG, which differ by two bases at their 3 ' ends (Figure 3A, lane 1). From a second aliquot, pools were made by 25 (lane 2) or 50 PCR cycles (lane 3 ), and then DD was applied as for the unamplified samples. The three patterns were very similar, indicating that pool formation did not distort the original distribution of double-stranded cDNAs and suggesting that cDNAs were proportionally amplified even when 50 PCR cycles preceded DD. The band intensities from the amplified pools were stronger than those obtained without amplification. As a control, bands were not produced if the RT step was omitted, showing that mRNAs were responsible (data not shown).

To further test the procedure for reproducibility, DD was performed using pools amplified with three different polymerases. Identical DNA fragment patterns were obtained with each primer combination. This result excludes the possibility that the cDNA pool is an artifact of the highly active rTth polymerase (data not shown). Additionally, we prepared 5 reverse transcriptase reactions from $\mathrm{LNCaP}$ and 5 from Jurkat cells (10000 cells). Then second-strand synthesis and amplification reactions were performed individually, creating 5 LNCaP 3' cDNA pools and 5 Jurkat pools. Using these $3^{\prime}$ cDNA pools for $\mathrm{DD}$, all $5 \mathrm{LNCaP}$ pools produced identical patterns, as did the 5 Jurkat cell cDNA pools (data not shown). This experiment gave further evidence for the reproducility of the cDNA pool by this amplification procedure, indicating that these DNA fragments are not the result of random amplification or primer dimerization.

We next investigated whether degenerate cDNA pools preserve similarities and differences between samples derived from different RNAs. LNCaP (Figure 3B, lane 1), PSA-positive WBC (lane 2) and PSA-negative WBC (lane 3) RNAs were used to prepare cDNA pools, which were then processed for DD using specific primers. The polyacrylamide gel patterns showed that most bands were common to all three samples. These equally amplified bands indicate that the method conserves the original mRNA similarities in the samples. As expected, some bands specific for a cell type also were found, as for the two PSA-positive specimens (Figure 3B, lanes 2 and 3, arrowhead). Using the indicated bands

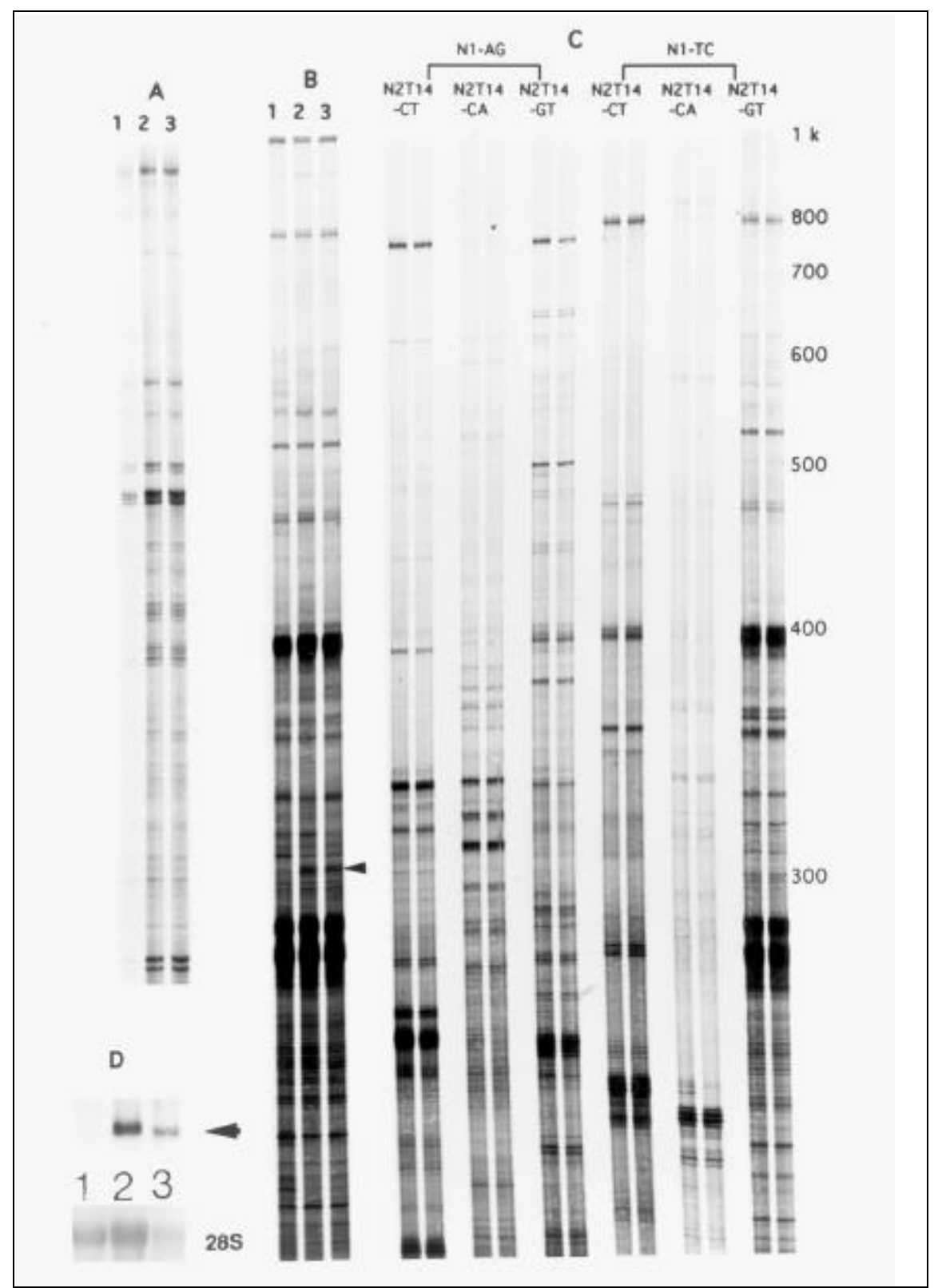

Figure 3. The degenerate cDNA library is suitable for differential display. (A) Degenerate primers equally amplify the total cDNA population, as seen on differential display. LNCaP cell RNA $(0.1 \mu \mathrm{g})$ was used for first-strand then second-strand cDNA synthesis to create an unamplified cDNA pool (lane 1) (Figure 1, Steps 1 and 2). Then a pool was constructed with the H-T14 and DP256 primers (lane 2). After purification on a QIAquick column, a sample was re-amplified with the degenerate primers (lane 3). All these samples were then processed for DD, using N1-TG and N2-T14GT nested primers. (B) cDNA libraries can conserve differences between samples. LNCaP (lane 1), PSA-positive (lane 2) and PSA-negative WBC (lane 3) RNAs were the sources for three degenerate cDNA libraries. These DNA pools were tested by DD, using N1-CC and N2-T14GT primers. (C) The degenerate cDNA library can function as a template for differential display. A library prepared from LNCaP cell RNA was tested with different primer combinations in differential display. The primer combinations are indicated at the top of the figure. (D) A displayed DNA fragment shows differential expression on a Northern blot. The band shown by an arrowhead on Panel B was excised and used as a probe for Northern hybridization. Lanes 1, 2 and 3 are the same as on Panel C. The 28S rRNA represents loading controls. 
to make a probe, a Northern blot confirmed that the corresponding mRNA was specific to LNCaP cells (Figure 3D, lanes 2 and 3) and was not visible in lane 1 , although the total RNA was comparable with lane 2 or even more than lane 3 . These results show that differences in DD expression levels are preserved in 3 '-end cDNA pools and also show that the pools contains sense, reproducible DNA fragments.

To test the general application of DD performed from amplified pools, we applied six combinations of primer pairs to a LNCaP cDNA pool (Figure 3C). Each primer pair displayed a unique pattern, indicating that each amplified a different subgroup of cDNAs. The bands were fewer than with standard DD, and their average size was longer when compared with the standard DD. Additional nested primers with one or three selecting bases were also investigated and showed similar quality DD patterns (data not shown). Therefore a relatively limited number

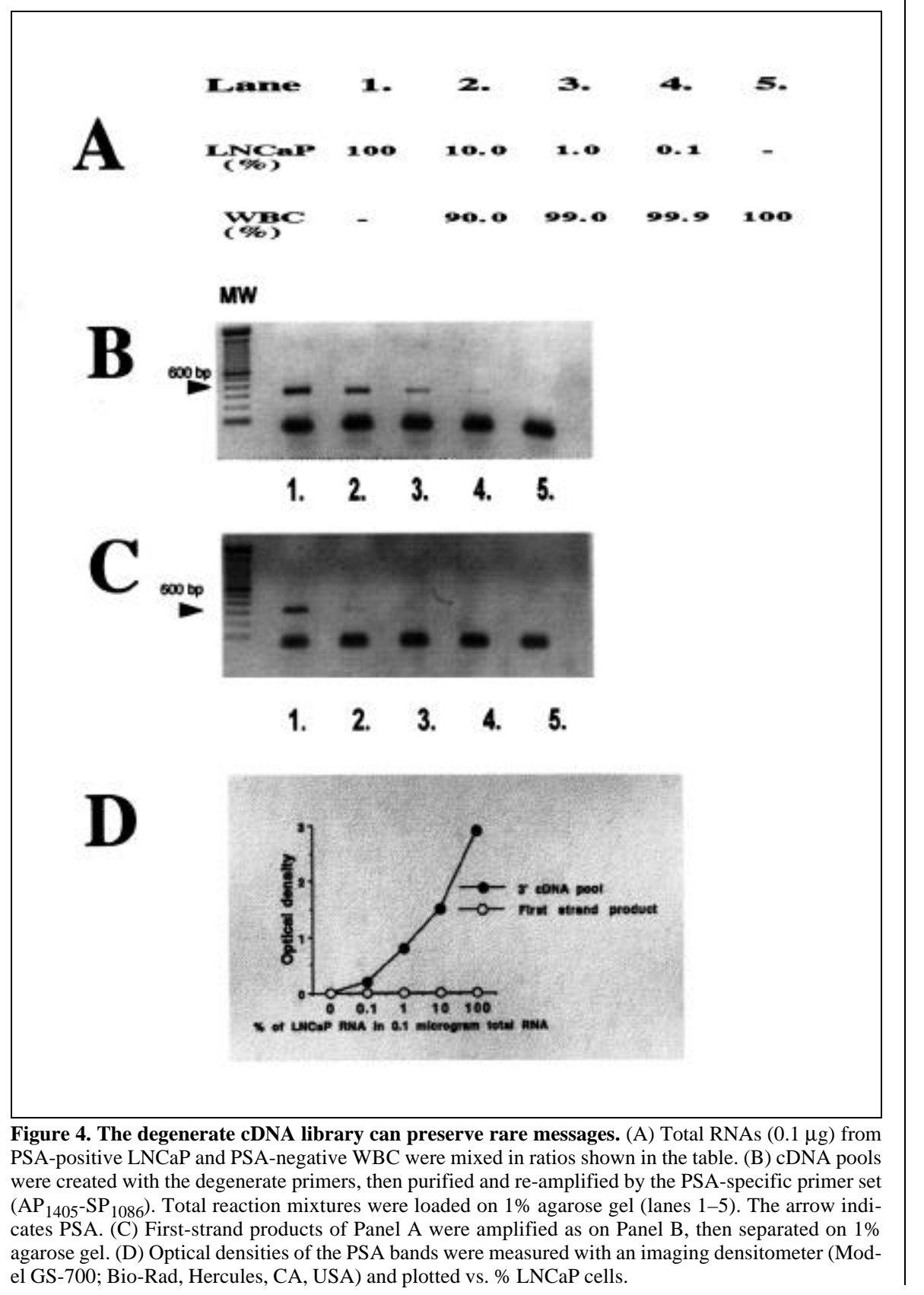




\section{Research Reports}

of primer sets should exhibit nearly the entire cDNA population, as with conventional DD.

DD patterns were also compared when the RT reaction or $3^{\prime}$ cDNA pools of $\mathrm{LNCaP}$ and WBC cells were used as starting material. The fragments made directly from reverse-transcribed, single-strand cDNAs or from the pools were similar in numbers and were similarly arranged. Some identical bands were found in the equivalent samples, but the majority were different for pool-DD or for RT-DD. This observa- tion is reasonable, because different templates (e.g., full-length, first-strand products vs. 3' ends of genes) were used (data not shown).

\section{Sensitivity of Detection of $3^{\prime}$ cDNA from a Pool}

The original DD utilized 10-100 $\mu \mathrm{g}$ of total RNA for extensive analysis (8). To determine sensitivity by which a rare message can be detected if a $3^{\prime}$ cDNA pool is constructed, we used PSA mRNA as a marker. We mixed

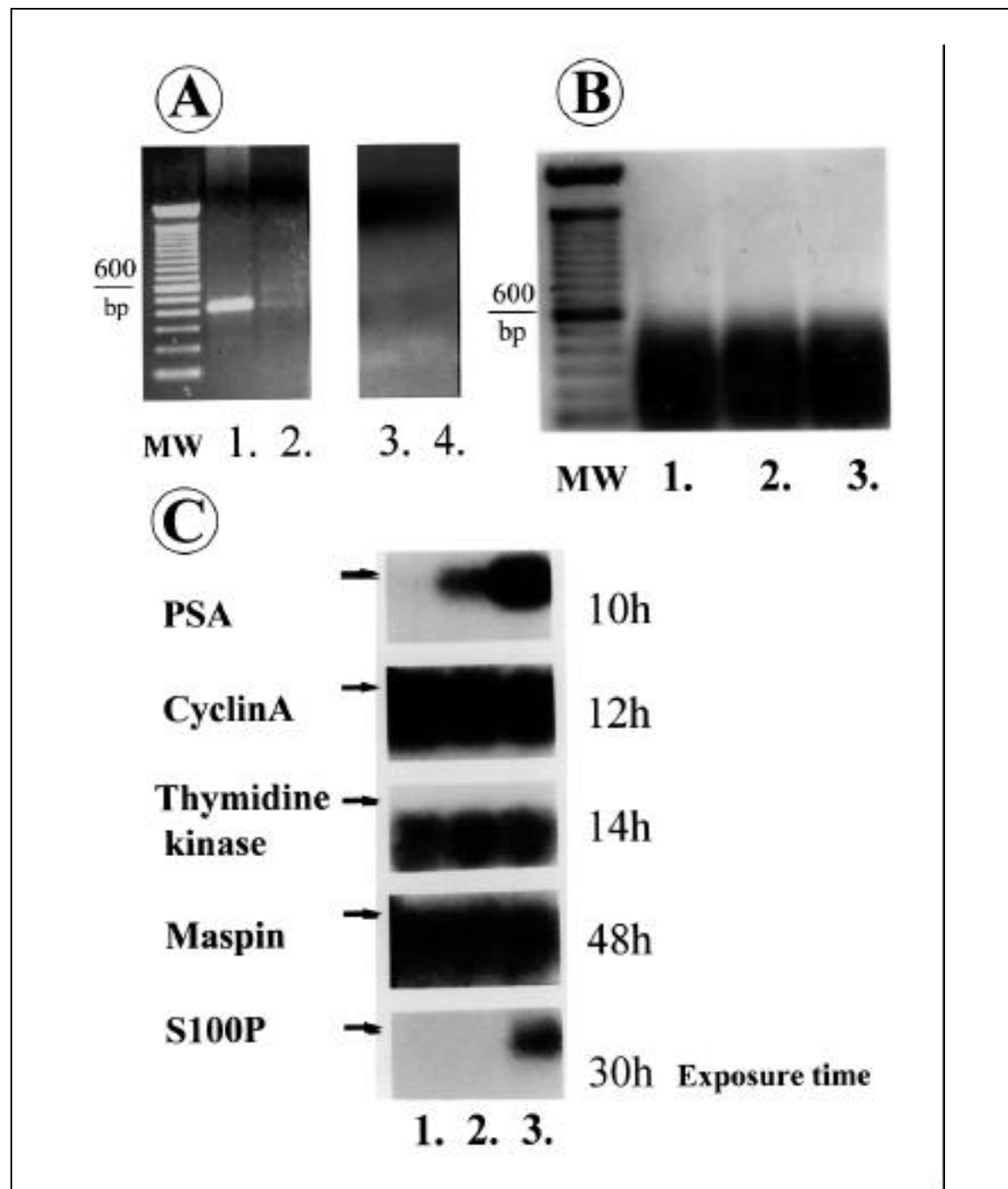

Figure 5. The degenerate cDNA library contains sense DNA fragments and serves as a template for specific primer amplification. (A) LNCaP first-strand product (lanes 2 and 4) and a 3'-end cDNA library (lanes 1 and 3) were amplified with specific primers for maspin (lanes 1 and 2) and HSIX genes (lanes 3 and 4). In all reactions, the same RT reaction products served as template for pool and for primer-specific amplifications. The PCR profiles from each different primer pair were the same between RT and pool samples. (B) 3' cDNA pools made from PSA-negative WBC (lane 1), PSA-positive WBC (lane 2) and LNCaP cell RNA, using the standard protocol. (C) Southern hybridization of Panel B samples. DNA probes shown on left side, exposure time on the right side of the blots. 600-bp molecular weight marker is indicated by the arrowhead.

$100 \%, 10 \%, 1 \%, 0.1 \%$ and $0 \mathrm{LNCaP}$ cell RNA with PSA-negative WBC RNA, keeping the total RNA constant at $100 \mathrm{ng}$ (Figure 4A). Pools were prepared according to Figure 1. To identify the PSA cDNA in these pools, PSAspecific primers that do not show any similarity to the initial primers were used in a second PCR (see Materials and Methods). The PCR products also were analyzed by Southern blot hy-

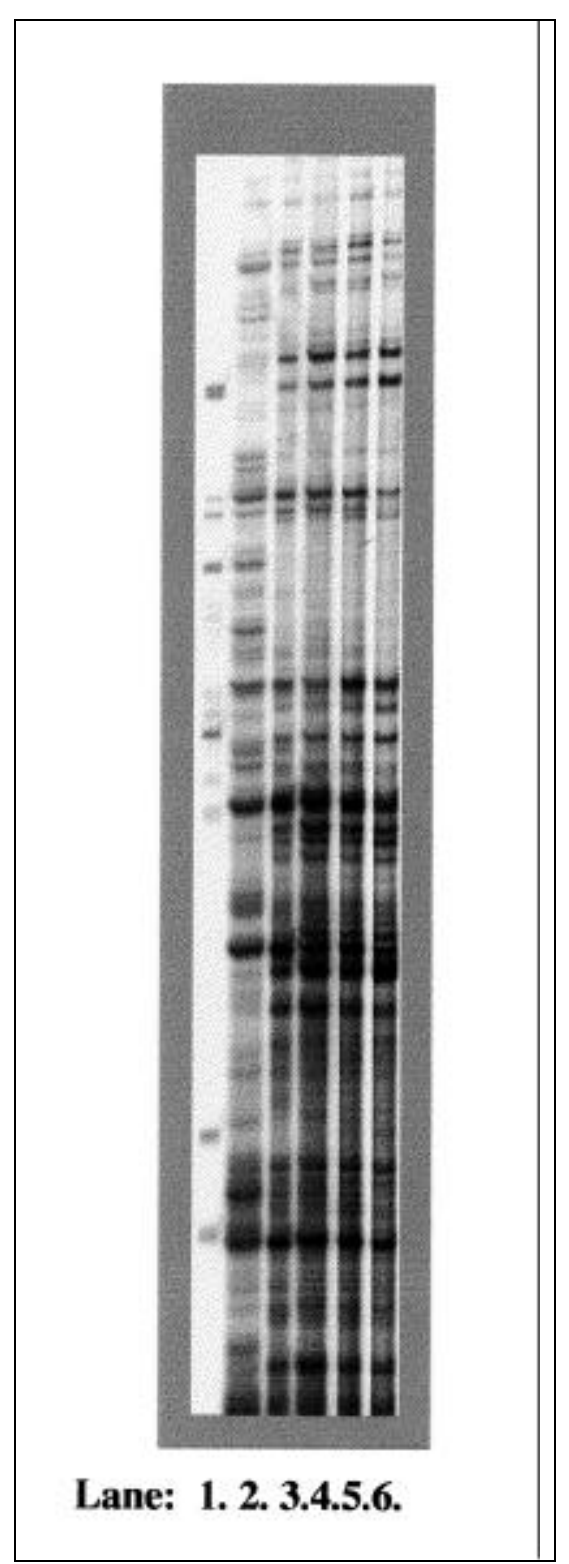

Figure 6. Reproducible DD pattern can be observed from $10^{4}$ cells. $10^{2}, 10^{3}, 10^{4}, 10^{5}$ and $10^{6}$ LNCaP cells (lanes $1-5$ ) or $0.1 \mu \mathrm{g} \mathrm{LNCaP} \mathrm{RNA}$ (lane 6) were processed by the standard procedure to get 3' cDNA pools. Samples then were used for DD by HT11-C and H-AP4 primers, supplied by the RNAimage Kit 1 . 


\section{Research Reports}

bridization. PSA cDNA was not detected with the PSA-negative WBC sample (Figure 4B, lane 5), and a positive signal was observed with even the smallest amount of LNCaP RNA (0.1 ng) (Figure 4B, lane 4) at the expected molecular weight (ca. $350 \mathrm{bp}$ ). Since PSA is moderately expressed in LNCaP cells $\left(10^{3}\right.$ copies/cell), the data represent about 1 PSA mRNA per cell at the
$0.1 \%$ ratio. A similarly low-abundance, short-transcript S100P was also well represented (see below).

For comparison, the $100 \% \mathrm{LNCaP}$ cell sample was required to detect PSA cDNA after direct RT, using the same primers for amplification (Figure 4C). Quantitation of PSA fragments in these samples showed a nonlinear dose dependence; however, the pool preserved rare messages. These results obtained without pool formation, show that firststrand DNA carryover is not responsible for the final signal.

Specific products other than PSA were also identified, showing that this cDNA pool contains representative $3^{\prime}$ fragments. Maspin and HSIX genes were chosen because of their different expression levels. A pool made from LNCaP RNA served as a template for specific amplification by primers that bind about $600 \mathrm{bp}$ from the poly(A) tails of their mRNAs (Figure 5A, lanes 1 and $3)$. As a control, $2 \mu \mathrm{L}$ of the RT reaction product were also amplified using the same conditions (Figure 5A, lanes 2 and 4). Maspin, which is very weakly expressed in LNCaP cells (12), was clearly detected from the pool, but only very weakly in the RT sample, while the muscle-specific HSIX gene (2) was not amplified from either the pool or the first-strand product of the WBC.

To confirm these results, we detected these products by applying Southern blot hybridization. Three $3^{\prime}$ cDNA pools were created from PSA-negative WBC, PSA-positive WBC and LNCaP cells that express both PSA and S100P. The total pools were run on a $1.2 \%$ agarose gel (Figure 5B), transferred and then probed (Figure 5C) with the indicated labeled DNA. Cyclin A, thymidine kinase and maspin, which are all present in these three cell lines, were detected in these pools. The different PSA expression levels in the two WBC samples were conserved in the pools. Similarly, pool specificity was observed for $\mathrm{S} 100 \mathrm{P}$, which is characteristic of LNCaP cells.

\section{A Usable 3' cDNA Pool Can Be Made from 1000 Cells}

The sensitivity of the method with regard to the intial number of cells was evaluated with increasing numbers of LNCaP cells $\left(1,10^{1}, 10^{2}, 10^{3}, 10^{4}, 10^{5}\right.$ and $\left.10^{6}\right)$. RNA was isolated using the RNeasy kit (Qiagen) in the presence of $20 \mu \mathrm{g}$ glycogen and immediately copied with reverse transcriptase. The $3^{\prime}$ cDNA pools were prepared according to Figure 1 and were then amplified with PSA-specific primers. PSA cDNA from only $1000 \mathrm{LNCaP}$ cells was clearly and reproducibly detected by ethidium 
bromide staining, but it was only occasionally and weakly visible with lower cell numbers. We note that it is unclear whether glycogen protects RNA during the RNeasy isolation procedure.

We also compared the DD patterns of $3^{\prime}$ cDNA pools prepared from different numbers of cells (Figure 6). We found a reproducible DD pattern with over $10^{4}$ cells (Figure 6, lanes, 3-6), and this pattern was the same between different preparations. Interestingly, cDNA pools prepared from a lower numbers of cells (1000 cells in lane 2 ) showed a higher band density DD than from higher numbers $\left(10^{4}\right)$. Reproducibility of this pattern with small cell numbers between experiments has not been studied.

For comparison, in a similar experiment performed with RNA from mixed LNCaP cells and WBC (see above), the resulting PSA band was detected with 200 times more sensitivity; it was seen with the $0.1 \%$ LNCaP cell sample, which was calculated to contain only 5 LNCaP cells (Figure 4B, lane 4). This large sensitivity difference between cells and RNA may derive from protection against degradation by the excess RNA $(0.1 \mu \mathrm{g})$ from the WBC or against loss of cells or RNA during isolation.

\section{DISCUSSION}

In summary, the majority of the transcribed genome can be screened from a 3 '-end cDNA pool by using DD. The results presented here suggest that our method of starting with one pair of degenerate primers produces a pool containing most $3^{\prime}$-end cDNAs and preserves the original frequency of different mRNAs. This amplified cDNA pool can be used for DD, applying large numbers of primer pairs, and thereby increases the number of expressed genes that can be identified from a small specimen. The cDNA pool is highly sensitive; the 20 ng of RNA initially obtained from 1000 cells are sufficient for virtually unlimited DD reactions. Without synthesis of this cDNA pool, this number of cells would permit only a few DD reactions. Rare messages are amplified, and so are more readily detectable from an amplified pool. Since each DD pattern produced with a spe- cific primer pair from a given cDNA pool represents a subset of the original mRNAs, they permit the identification of many differentially expressed genes.

Another possible application of the amplified cDNA pool is to perform amplification with gene-specific primers to detect a known gene in a small amounts of RNA. The data suggest that the cDNA pool can provide starting material for specific amplification when the sample amount is not sufficient for detection by direct RT-PCR. These pools very sensitively preserved the differential expression of known genes and so may be useful when sufficient RNA (up to $20 \mu \mathrm{g}$ ) is not available for Northern blot hybridization. The pool technique is also useful for making a 3' probe from the isolated DD. These probes can be used also to find full-length sequences and to detect the corresponding mRNAs in material in which they are scarce. Finally, the library construction is not time-consuming or expensive, thus it could easily be adapted to produce cDNA for other screening techniques.

\section{ACKNOWLEDGMENTS}

S. Zhao and G. Molnar contributed equally to this paper. This work was aided by Grant No. CA61232 from the National Institutes of Health and funds from Ciba Corning Diagnostics, Inc.

\section{REFERENCES}

1.Averboukh, L., P. Liang, P.W. Kantoff and A.B. Pardee. 1996. Regulation of S100P expression by androgen. Prostate 29:350-355.

2.Boucher C.A., N. Carey, Y.H. Edwards, M.J. Siciliano and K.J. Johnson. 1996. Cloning of the human SIX1 gene and its assignment to chromosome 14. Genomics 33:140-142.

3.Brady, G. and N.N. Iscove. 1993. Construction of cDNA libraries from single cells. Methods Enzymol. 225:611-622.

4.Diatchenco, L., Y.-F.C. Lau, A.P. Campbell, A. Chenchik, F. Moqadam, B. Huang, S. Lukyanov, K. Lukyanov et al. 1996. Suppression substractive hybridization: a method for generating differentially regulated or tissue-specific cDNA probes and libraries. Proc. Natl. Acad. Sci. USA 93:6025-6030.

5.Fazely, F., B.J. Dezube, J. Allen-Ryan, A.B. Pardee and R.M. Ruprecht. 1991. Pentoxifylline (Trental) decreases the replication of the human immunodeficiency virus type 1 in human peripheral blood mononuclear cells and in cultured T cells. Blood 77:1653-1656.

6.Froussard, P. 1992. A random-PCR method (rPCR) to construct whole cDNA library from low amounts of RNA. Nucleic Acids Res. 20:2900.

7.Liang, P., L. Averboukh and A.B. Pardee. 1993. Distribution and cloning of eukaryotic mRNAs by means of differential display: refinements and optimizations. Nucleic Acids Res. 21:3269-3275.

8.Liang, P. and A.B. Pardee. 1992. Differential display of eukaryotic messenger RNA by means of the polymerase chain reaction. Science 257:967-971.

9.Liang, P. and A.B. Pardee. 1997. Differential Display Methods and Protocols. Humana Press, Totowa, NJ.

10.Maniatis, T., E.F. Fritsch and J. Sambrook. 1982. Extraction, purification and analysis of mRNA from eukaryotic cells, p. 187-211. Molecular Cloning: A Laboratory Manual. CSH Laboratory Press, Cold Spring Harbor NY.

11.Maniatis, T., E.F. Fritsch and J. Sambrook. 1982. Southern transfer, p. 382-390. Molecular Cloning: A Laboratory Manual. CSH Laboratory Press, Cold Spring Harbor NY.

12.Sheng, S., J. Carey, E.A. Seftor, L. Dias, M. J.C. Hendrix and R. Sager. 1996. Maspin acts at the cell membrane to inhibit invasion and motility of mammary and prostatic cancer cells. Proc. Natl. Acad. Sci. USA 93:1166911674

13.Zhao, S., S.L. Ooi and A.B. Pardee. 1995 New primer strategy improves precision of differential display. BioTechniques 18:842850 .

Received 26 June 1997: accepted 19 December 1997.

Address correspondence to:

Dr. Arthur B. Pardee

Dana-Farber Cancer Institute

44 Binney Street

Boston, MA 02115, USA

Internet: pardee@mbcrr.harvard.edu 